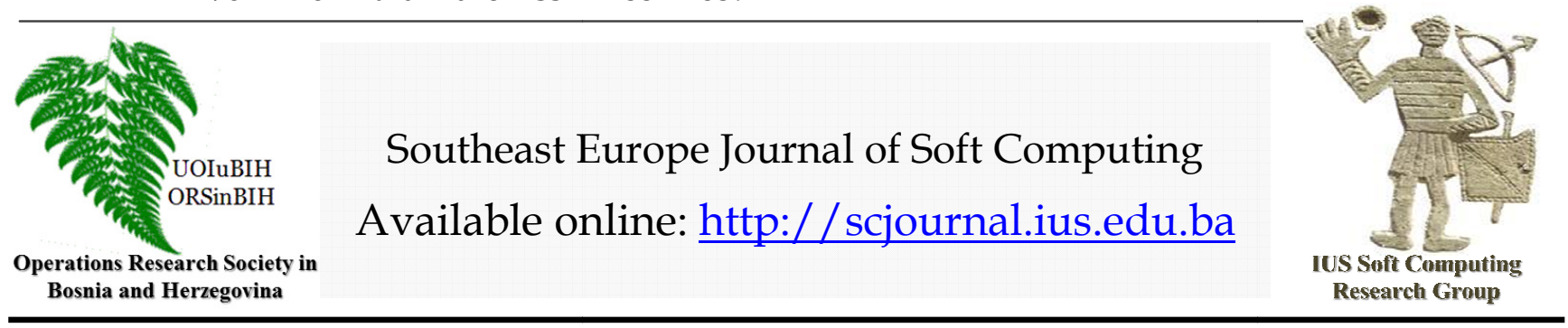

\title{
The Gut-Brain Axis in Foetal-Maternal Relationship
}

\section{Mahmutovic}

Faculty of Engineering and Natural Sciences, International University of Sarajevo International University of Sarajevo, Hrasnicka Cesta 15, Ilidža 71210 Sarajevo,

Bosnia and Herzegovina

lhalilovic@student.ius.edu.ba

\section{Article Info}

Article history:

Article received on 20 January 2018 Received in revised form 1 February 2018

Keywords:Microbiome, microbiota, pregnancy, gut-brain axis

\begin{abstract}
Gut-brain axis (GBA) represents a bidirectional communication between the central nervous system (CNS) and gastrointestinal (GI) tract. Microbiota found in GI tract has beneficial relationship with their host and can affect the brain, behavior. Studies performed on animals suggest that any change in the composition of microbiota might cause alternations in behavior. In the same way, changes in behavior such as stress, showed to affect the microbiota. Moreover, the composition of the maternal microbiome in pregnancy is known to adversely influence neonatal and infant health and preterm birth. Mother's microbiome is inherited from the mother to children.
\end{abstract}

\section{INTRODUCTION}

Gastro intestinal (GI) microbiota is functionally diverse with crucial role in proper functioning of the entire body. Human gut microbiota consists of 100 trillion microorganisms including more than one thousand different species of bacteria, fungi, viruses, and other life forms living in our intestinal linings. This is 10 -fold greater than the number of human cells. The collective genome of the gut microbiota is estimated to contain more genes than the human genome (Evernesel and Ceylan, 2015; Mu et al., 2016).

Recent advances in technology has expanded our knowledge of the various functions of the gut microbiota made of different species of beneficial and non-beneficial microbes. For instance, Lactobacillus and Bifidobacterium species, known as beneficial microbes, are widely used as probiotics to improve intestinal homeostasis. However, Akkermansiamuciniphila and Bacteroides thetaiotaomicron, are well-known for their role in the mucin degradation (Kelly et al., 2015; Mu et al., 2016).

Having a healthy balance of microbiota composition results in intestine homeostasis. However, the microbial community can be affected by many environmental factors and host-related factors such as unhealthy diet, smoking, alcohol consumption, pollution, stress and others (Mayer et al., 2015).

Pregnancy is an amazing biological process that involves simultaneous changes in many physiological systems to help the development of healthy offspring. These changes include hormonal changes, immune system modulation, weight gain and others, which must all be coordinated to sustain the health of both the mother and the offspring (Dunlop et al., 2015; Nuriel-Ohayon et al., 2016). One of these changes also includes dramatic alternations in microbiome composition that happen during gestation 
period. When studying the role of the microbiota in pregnancy, it is important to think of the stage at which the crucial interaction between the host and its microbes begins. More precisely, it is important to consider when does the developing fetus first come in contact with microbes. Over 100 years ago, it was hypothesized that humans are born germ free, without presence of any microbe. Even though this is still the belief of many physicians, numerous evidences now cast doubt on this hypothesis, and propose that a bacterial presence already exists in the feto-placental unit (Nuriel-Ohayon et al., 2016).

In this review, we will outline the microbial changes that occur in the pregnant female, as well as provide an overview of the gut-brain axis in the foetal-maternal relationship. We hypothesize that a healthy balance of microbiota composition is crucial for development, pregnancy maintenance, and the early childhood. Moreover, we suggest that the alternations in the human microbiome are closely associated to the host's physiological status, environmental factors and behavior which could be inherited from mother to the offspring. Therefore, realizing the role of the microbiome throughout pregnancy and early development is of great importance for suggesting new research opportunities and even developing new therapeutic approaches.

\section{THE HUMAN GUT MICROBIOTA}

The term microbiome refers to all microorganisms and their genetic material that inhabit our body whereas the microbiota refers to the unique populations of microorganisms present in the body's various ecosystems such as the gut microbiota, skin microbiota, placental microbiota, vaginal microbiota and other (Clemmensen et al., 2017; Mayer et al., 2015). Besides having role in GI disorders, bacteria in our guts secrete a large number of neurochemicals our neurons use to communicate and regulate mood. Variations in the microbial community can affect behavior whereas changes in behavior can alter the composition of the gut microbiota (Mu et al., 2016).

For instance, Lactobacillus and Bifidobacterium synthesize gamma-aminobutyric acid (GABA) from monosodium glutamate (Yunes et al., 2016) which is relevant for serotonin production.Also, it is known thatEscherichia coli, Bacillus and Saccharomyces produce norepinefrin whereas Candida, Streptococcus, Escherichia and Enterococcus produce serotonin. In addition, Bacillus and Serratiaspecies produce dopamine, a neurotransmitter related to happiness.Members of the genus Bifidobacterium, specifically Bifidobacterium infantis, are among the first microbes that colonize human GI tract is (O'Callaghan et al., 2016). These bacteriaare also taken as a probiotic, due to its known role in increasing serotonin levels. In several studies, upon treatment of model organisms with $B$. infantis, increased plasma tryptophan levels were observed, that resulted in increased serotonin levels (Evernesel and Ceylan, 2015).Moreover, B. longum has anti-inflammatory, anti-carcinogenic, and antimutagenic properties and may protect colon cancer development.

In addition to this, changes in microbiota composition might be induced during pregnancy as well, as described by various studies. Gohir at al. reported that microbiota composition changes during the pregnancy in a way that specific genere such as Akkermansia, Clostridium, Bacteroides and Bifidobacteriumareare significantly increased during pregnancy compared to non-pregnant females (Gohir et al., 2015).

More than 50 microbial species considered non-pathogenic are found in the vaginal microbiome and are considered to be non-pathogenic (Dunlop et al., 2015). In the reproductive age, the vaginal microbiome varies widely. The healthy vaginal microbiome plays a role in preventing bacterial vaginosis, sexually transmitted infections, urinary tract infections, and HIV (Dunlop et al., 2015). The protective role of vaginal microbiome is generally credited to the lactic acid-producing bacteria, mainly Lactobacillus species which can disadvantage virulent microbes (Boskey at al., 2001).

\section{GUT MICROBIOTA CHANGES DURING PREGNANCY}

Increase in the bacterial load and extreme alterations in the composition of gut microbiota are observed in the healthy pregnancy (Nuriel-Ohayon et al., 2016). Similar gut microbial composition to that of healthy, non-pregnant women is present in the first trimester of pregnancy. However, from the first to the third trimester, the gut microbiota is changed a lot and is characterized by an increased abundance of members of the Actinobacteria and Proteobacteria phyla. These changes are coupled with weight gain, insulin isn sensitivity, and higher levels of fecal cytokines, reflecting inflammation and other.

In order to investigate the roles of the third trimester microbial profiles, researchers used fecal transplantation to introduce samples of first- and third-trimester fecal microbiotas to germ-free (GF) mice, and to examine their physiological effects. The third trimester gut-microbiota in GF mice caused significant weight increase, insulin resistance development, and a greater inflammatory response compared to the first-trimester transplanted mice (Koren et al., 2012). These findings are the proof that the microbial composition can lead to enormous changes in host immunology and metabolism. However, such bacterial composition observed in the third trimester of pregnancy contributes to a healthy pregnancy, and proper fetal development (Romano-Keeler et al., 2014).

The gut microbiota during pregnancy is influenced by internal and environmental factors, primarily by diet (Gohir et al., 2015) and/or medicines (Langdonet al., 2016; Rodríguez et al., 2015). The effects of antibiotics administered during pregnancy on the microbiome have 
been studied in rats (Khan et al., 2016). Researches showed that antibiotic consumption increased the fecal presence of Proteobacteria and Enterobacter, while reducing the presence of Firmicutes and Lactobacillus (Khan et al., 2016; Rodríguez et al., 2015).

\section{COMPOSITION OF GUT MICROBIOTA AFFECTS BRAIN FUNCTION AND BEHAVIOUR}

Besides being home to a large ecosystem of micro organisms, the GI also contains approximately 50,000 extrinsic and 100 million intrinsic sensory afferent neurons, all of which function in proximity to the trillions of microorganisms housed in the intestinal lumen (Cowan et al., 2017; Dunlop et al., 2015). As reviewed in other articles, human gut microbiota might play role in behavior and mental activity. Potential mechanisms influencing the microbiota on the central nervous system (CNS) is through the vagus nerve that represents a direct connection between the brain and stomach. Changes in microbial content, immune stimulation, neural pathways tryptophan metabolism, gut hormonal response, and bacterial metabolites can all have effect on human brain (Evernesel and Ceylan, 2015).

However, upon the exposure to stress, some changes in the microbiota composition were observed. Those changes mainly influence production of GABA and serotonin neurotransmitter (Pokusaeva et al., 2016).

\section{FOETAL-MATERNAL RELATIONSHIP}

Based on many studies, mother's physiological state influences the composition of the foetal microbiome formation. If mother was exposed to stress, medicines, bad diet, alcohol or drug usage during pregnancy, might cause extreme alternation in the microbiome composition by allowing bad bacterial colonies to be in advantage over the beneficial colonies. In addition, exposure to abovementioned factors after the birth, in postnatal period might have the same effect on the offspring's microbiome.

Some researches done on this topic claim that postnatal stress caused by the separation of babies from their mother changed the microbiota showed by decreased levels of probiotic including Bifidobacterium and Lactobacillus (Evernesel and Ceylan, 2015;Kelly et al., 2015). Stressful early-life events might be strongly related to the development of depression later in life (Kelly et al., 2015). Study done by Kelly and colleagues (2015) showed that reduced fecal Lactobacillus numbers three days postseparation of pups from their mother were correlated with stress-related behaviors (Kelly et al., 2015).

Most strikingly, certain types non-absorbable antibiotics given to pregnant mice changed offspring behavior. Pups exhibited low locomotor activity at 4 weeks of age, and less investigative behavior in central regions at 4 and 8 weeks of age (Tochitani et al., 2016).

Moreover, it has been hypothesized that microbial exposure during pregnancy may be significant for preventing allergic disease in the offspring (Abrahamsson et al., 2015). Accordingly, in a study in which mothers with allergic disease received probiotics during the last 2 months of pregnancy and the first 2 months of lactation, a reduced risk for allergic reaction in the offspring was observed compared to placebo treatment (Rautava et al., 2012).

\section{CONCLUSIONS}

Gut-Brain axis is bidirectional, creating network between the gut microbiota and the brain, which may unravel a new way for better functioning of brain and gut in benefit of human health. Biological changes that occur during pregnancy are likely to alter the microbial community in the gut which might be transferred to pops. The stress in pregnant mothers also might impact the composition of microbiota in the gut. However, treatment with beneficial bacteria might increase levels of serotonin and GABA, two important neurotransmitters. Additional research need to be done on this topic in order to better understand gutbrain communication and its role in the gut-brain axis.

\section{REFERENCES}

Abrahamsson, T., Wu, R., \& Jenmalm, M. (2014). Gut microbiota and allergy: the importance of the pregnancy period. Pediatric Research, 77(1-2), 214-219. http://dx.doi.org/10.1038/pr.2014.165

Boskey, E., Cone, R., Whaley, K., \& Moench, T. (2001). Origins of vaginal acidity: high $\mathrm{d} / \mathrm{l}$ lactate ratio is consistent with bacteria being the primary source. Human Reproduction, 16(9), 1809-1813.

http://dx.doi.org/10.1093/humrep/16.9.1809

Bravo, J., Forsythe, P., Chew, M., Escaravage, E., Savignac, H., \& Dinan, T. et al. (2011). Ingestion of Lactobacillus strain regulates emotional behavior and central GABA receptor expression in a mouse via the vagus nerve. Proceedings Of The National Academy Of Sciences, 108(38), 16050-16055.

Clemmensen, C., Müller, T., Woods, S., Berthoud, H., Seeley, R., \& Tschöp, M. (2017). Gut-Brain Cross-Talk in Metabolic Control. Cell, 168(5), 758-774.

Cowan, C., Hoban, A., Ventura-Silva, A., Dinan, T., Clarke, G., \& Cryan, J. (2017). Gutsy Moves: The Amygdala as a Critical Node in Microbiota to Brain Signaling. Bioessays, 40(1), 1700172. http://dx.doi.org/10.1002/bies.201700172

Dominguez-Bello, M., Blaser, M., Ley, R., \& Knight, R. (2011). Development of the Human Gastrointestinal Microbiota and Insights From High-Throughput Sequencing. Gastroenterology, 140(6), 1713-1719. 
Dunlop, A., Mulle, J., Ferranti, E., Edwards, S., Dunn, A., \& Corwin, E. (2015). Maternal Microbiome and Pregnancy Outcomes That Impact Infant Health. Advances In Neonatal Care, 15(6), 377-385. http://dx.doi.org/10.1097/anc.0000000000000218

Gohir, W., Whelan, F., Surette, M., Moore, C., Schertzer, J., \& Sloboda, D. (2015). Pregnancy-related changes in the maternal gut microbiota are dependent upon the mother's periconceptional diet. Gut Microbes, 6(5), 310-320. http://dx.doi.org/10.1080/19490976.2015.1086056

Ho, J., Chan, G., \& Li, J. (2015). Systemic effects of gut microbiota and its relationship with disease and modulation. BMC Immunology, 16(1).

Kadurugamuwa, J., Sin, L., Yu, J., Francis, K., Kimura, R., Purchio, T., \& Contag, P. (2003). Rapid Direct Method for Monitoring Antibiotics in a Mouse Model of Bacterial Biofilm Infection. Antimicrobial Agents And Chemotherapy, 47(10), 3130-3137.

Langdon, A., Crook, N., \& Dantas, G. (2016). The effects of antibiotics on the microbiome throughout development and alternative approaches for therapeutic modulation. Genome Medicine, 8(1). http://dx.doi.org/10.1186/s13073016-0294-z

Maurice, C. F., Cl Knowles, S., Ladau, J., Pollard, K. S., Fenton, A., Pedersen, A. B., et al. (2015). Marked seasonal variation in the wild mouse gut microbiota. ISME J. 9, 2423-2434. doi: 10.1038/ismej.2015.53

Mayer, E., Tillisch, K., \& Gupta, A. (2015). Gut/brain axis and the microbiota. Journal Of Clinical Investigation, 125(3), 926-938.

Mayer, E., Tillisch, K., \& Gupta, A. (2015). Gut/brain axis and the microbiota. Journal Of Clinical Investigation, 125(3), 926-938.

Nuriel-Ohayon, M., Neuman, H., \& Koren, O. (2016). Microbial Changes during Pregnancy, Birth, and Infancy. Frontiers In Microbiology, 7. http://dx.doi.org/10.3389/fmicb.2016.01031

O'Callaghan, A., \& van Sinderen, D. (2016). Bifidobacteria and Their Role as Members of the Human Gut Microbiota. Frontiers In Microbiology, 7.http://dx.doi.org/10.3389/fmicb.2016.00925

Pokusaeva, K., Johnson, C., Luk, B., Uribe, G., Fu, Y., \& Oezguen, N. et al. (2016). GABA-producing Bifidobacterium dentium modulates visceral sensitivity in the intestine. Neurogastroenterology \& Motility, 29(1), e12904.

Rautava, S., Kainonen, E., Salminen, S., \& Isolauri, E. (2012). Maternal probiotic supplementation during pregnancy and breast-feeding reduces the risk of eczema in the infant. Journal Of Allergy And Clinical Immunology, 130(6), http://dx.doi.org/10.1016/j.jaci.2012.09.003
Rodríguez, J., Murphy, K., Stanton, C., Ross, R., Kober, O., \& Juge, N. et al. (2015). The composition of the gut microbiota throughout life, with an emphasis on early life. Microbial Ecology In Health \& Disease, 26(0). http://dx.doi.org/10.3402/mehd.v26.26050Khan, I., Azhar, E., Abbas, A., Kumosani, T., Barbour, E., Raoult, D., \& Yasir, M. (2016). Metagenomic Analysis of AntibioticInduced Changes in Gut Microbiota in a Pregnant Rat Model. Frontiers In Pharmacology, 7. http://dx.doi.org/10.3389/fphar.2016.00104

Romano-Keeler, J., \& Weitkamp, J. (2014). Maternal influences on fetal microbial colonization and immune development. Pediatric Research, 77(1-2), 189-195. http://dx.doi.org/10.1038/pr.2014.163

Yunes, R., Poluektova, E., Dyachkova, M., Klimina, K., Kovtun, A., \& Averina, O. et al. (2016). GABA production and structure of gadB / gadC genes in Lactobacillus and Bifidobacterium strains from human microbiota. Anaerobe, 42, 197-204. http://dx.doi.org/10.1016/j.anaerobe.2016.10.011 